\title{
вмJ Global Health Health extension workers improve tuberculosis case finding and treatment outcome in Ethiopia: a large-scale implementation study
}

\author{
Daniel G Datiko, ${ }^{1}$ Mohammed A Yassin, ${ }^{2}$ Sally J Theobald, ${ }^{3}$ Lucie Blok, ${ }^{4}$
} Sahu Suvanand, ${ }^{5}$ Jacob Creswell, Luis E Cuevas ${ }^{3}$

To cite: Datiko DG, Yassin MA, Theobald SJ, et al. Health extension workers improve tuberculosis case finding and treatment outcome in Ethiopia: a large-scale implementation study. BMJ Glob Health 2017;2:e000390. doi:10.1136/ bmjgh-2017-000390

Handling editor Seye Abimbola

DGD and MAY contributed equally.

Received 2 May 2017 Revised 14 September 2017 Accepted 6 October 2017

\section{(a) CrossMark}

${ }^{1}$ REACH Ethiopia, Hawassa, Ethiopia

${ }^{2}$ The Global Fund to Fight AIDS, Tuberculosis and Malaria, Geneva, Switzerland

${ }^{3}$ Faculty of Clinical Sciences and International Health, Liverpool School of Tropical Medicine, Liverpool, UK

${ }^{4}$ Royal Tropical Institute (KIT), Amsterdam, The Netherlands ${ }^{5}$ Stop TB Partnership, Geneva, Switzerland

Correspondence to Professor Luis E Cuevas; luis.cuevas@Istmed.ac.uk

\section{ABSTRACT}

Background Tuberculosis (TB) is a major cause of death in Ethiopia. One of the main barriers for TB control is the lack of access to health services.

Methods We evaluated a diagnostic and treatment service for TB based on the health extension workers (HEW) of the Ethiopian Health Extension Programme in Sidama Zone, with 3.5 million population. We added the services to the HEW routines and evaluated their effect over 4.5 years. $1024 \mathrm{HEWs}$ were trained to identify individuals with symptoms of TB, request sputum samples and prepare smears. Smears were transported to designated laboratories. Individuals with TB were offered treatment at home or the local health post. A second zone (Hadiya) with 1.2 million population was selected as control. We compared TB case notification rates (CNR) and treatment outcomes in the zones 3 years before and 4.5 years after intervention.

Results HEWs identified 216165 individuals with symptoms and 27918 (12\%) were diagnosed with TB. Smear-positive TB CNR increased from 64 (95\% Cl 62.5 to 65.8 ) to 127 (95\% Cl 123.8 to 131.2) and all forms of TB increased from 102 (95\% Cl 99.1 to 105.8$)$ to 177 (95\% Cl 172.6 to 181.0$)$ per 100000 population in the first year of intervention. In subsequent years, the smear-positive CNR declined by $9 \%$ per year. There was no change in CNR in the control area. Treatment success increased from $76 \%$ before the intervention to $95 \%$ during the intervention. Patients lost to follow-up decreased from $21 \%$ to $3 \%(p<0.001)$.

Conclusion A community-based package significantly increased case finding and improved treatment outcome. Implementing this strategy could help meet the Ethiopian Sustainable Development Goal targets.

\section{INTRODUCTION}

Tuberculosis (TB) is a leading cause of death among infectious diseases and is responsible for 1.8 million deaths each year. ${ }^{1}$ Over 4 million incident cases of TB worldwide fail to be diagnosed or linked into TB services every year ${ }^{1}$ and people with undetected TB have a high mortality and perpetuate disease transmission. Identifying and treating these individuals is crucial.
Key questions

What is already known about this topic?

- Tuberculosis disproportionally affects poor populations in urban slums and rural areas.

- Access to tuberculosis diagnostic and treatment services is a major barrier for these populations.

- An estimated 4.5 million individuals with tuberculosis do not reach the health services each year.

- Finding these 'missing' cases is an international priority for tuberculosis control.

What are the new findings?

- Female community health workers providing services for the diagnosis and treatment of tuberculosis at village level significantly increase the number of cases diagnosed in the rural areas.

- The proportion of women screened in this service was higher than in the health centres.

- The service increased the proportion of individuals who completed treatment, reaching an unprecedented $95 \%$.

- The proportion of patients lost to follow-up decreased to an unprecedented low level.

- After an initial peak in the number of cases, the case notification rate declined from this peak by $9 \%$ per year, suggesting a decline in transmission.

Recommendations for policy

- The study confirms that there is a large pool of individuals with tuberculosis who do not access health services.

- Countries attempting to increase the detection of tuberculosis in rural areas need to consider that the poor accessibility of services is a major barrier for tuberculosis diagnosis and treatment.

- The provision of services at community level increases case detection, treatment acceptance and treatment completion.

Although TB incidence has been declining since 2002, this decline has been slow, only achieving a reduction of $<2 \%$ per year and 


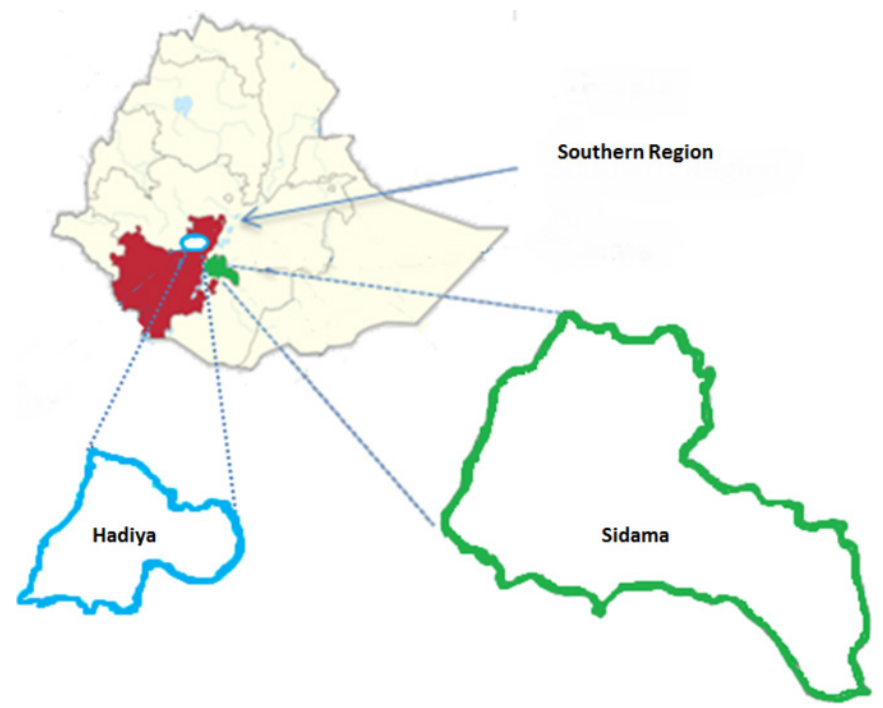

Figure 1 Map of the implementation (Sidama) and control (Hadiya) zones.

achieving the Sustainable Development Goals of ending the TB epidemic in $2030^{2}$ will require a paradigm shift in how we address TB. This would require concerted efforts and investment into innovative high-impact interventions that reach all populations and eliminate the barriers to access services. ${ }^{3}$ TB programmes have historically followed passive case finding approaches in which services wait for individuals with symptoms to visit health facilities, ${ }^{45}$ but symptoms of TB are non-specific and travelling to health facilities is costly and difficult in many settings, particularly in rural areas. ${ }^{67}$

Ethiopia has the third highest burden of TB cases in Africa with an estimated incidence of 207 cases (all forms) of TB per 100000 population. Despite a major expansion and decentralisation of diagnostic and treatment centres, the case detection is still $74 \%$ and it is estimated that the health system misses about 50000 people with incident TB each year. ${ }^{8}$

Ethiopia however is a country that is making considerable progress in improving its health system, which is partly due to the government launching the Ethiopian Health Extension Programme (HEP) in 2003 to increase population access to basic health services. The HEP trains and employs female village-based health workers, called health extension workers (HEW), to regularly visit households in their villages (called kebeles) to implement basic packages of healthcare. For this study, we added a diagnostic and treatment service for TB to their packages of service and evaluated the effects on a large-scale and over a long duration.

\section{METHODS}

This was a community-based intervention based in Sidama Zone, in the Southern Nations, Nationalities, and Peoples' Region (SNNPR) of Ethiopia. Sidama has 524 rural and 39 urban kebeles with a population of 3.5 million. Kebeles or villages are the smallest administrative unit of the country and have approximately 5000 population. The zone covers an area of $10000 \mathrm{~km}^{2}$ and the population is dispersed (figure 1). Transport facilities are limited and relatively expensive, making travel to health facilities challenging. All kebeles in Sidama were included in our study.

The intervention consisted of engaging the HEWs of the Ethiopian HEP and facilitating their linkage to laboratory services and treatment through a transport network using motorbikes and was implemented in partnership with the Sidama Zone Health Department and the SNNPR Health Bureau. Before the intervention, HEWs were expected to visit all the households in their kebeles fortnightly, to provide education for TB, counselling on treatment adherence and referred persons with presumptive TB to the health centres. However, few patients were identified and the uptake of referral was very low.

The intervention package is summarised in box and was described in detail in a previous publication ${ }^{9}$ (figure 2). In brief, all 1024 HEWs of the HEP in Sidama were trained by project and TB Programme staff in 5-day workshops to identify household members with symptoms suggestive of TB (mostly by asking if there were residents with persistent cough), request one sputum sample from symptomatic persons and learn how to prepare smears in the local health post. Smears were then transported from the village to a designated laboratory on motorbike by a HEW supervisor. Smears were examined using light smear microscopy. Persons with smear-positive TB were

Box Summary of the intervention package implemented in Sidama Zone

1. Capacity strengthening: training for health workers, HEWs and laboratory staff; provision of LED fluorescent microscopes to highvolume microscopy centres and GeneXpert for two centres.

2. Continuous advocacy, communication and social mobilisation (ACSM).

3. Fortnightly house-to-house visits by HEWs to identify residents with chronic cough, inform them how to produce sputum and prepare smears for smear microscopy. Xpert testing for children, HIV-positive individuals and patients who continued to be symptomatic after two smear-negative results.

4. Provision of communication facilities and transport. HEWs and project supervisors received airtime credits to facilitate communication. Supervisors had motorbikes to link the HEWs to TB diagnostic and treatment services.

5. Supervisors transported smears to microscopy centres, communicated test results, registered patients by proxy at health centres to release treatment and initiated treatment in the community. Patients chose to receive the treatment at home or at the kebele health post staffed by the HEWs.

6. HEWs and supervisors screened household contacts to identify and investigate symptomatic individuals, and to provide IPT for children, and followed people with smear-negative results.

HEW, health extension worker; IPT, isoniazid preventative therapy; LED, lightemitting diode; TB, tuberculosis. 


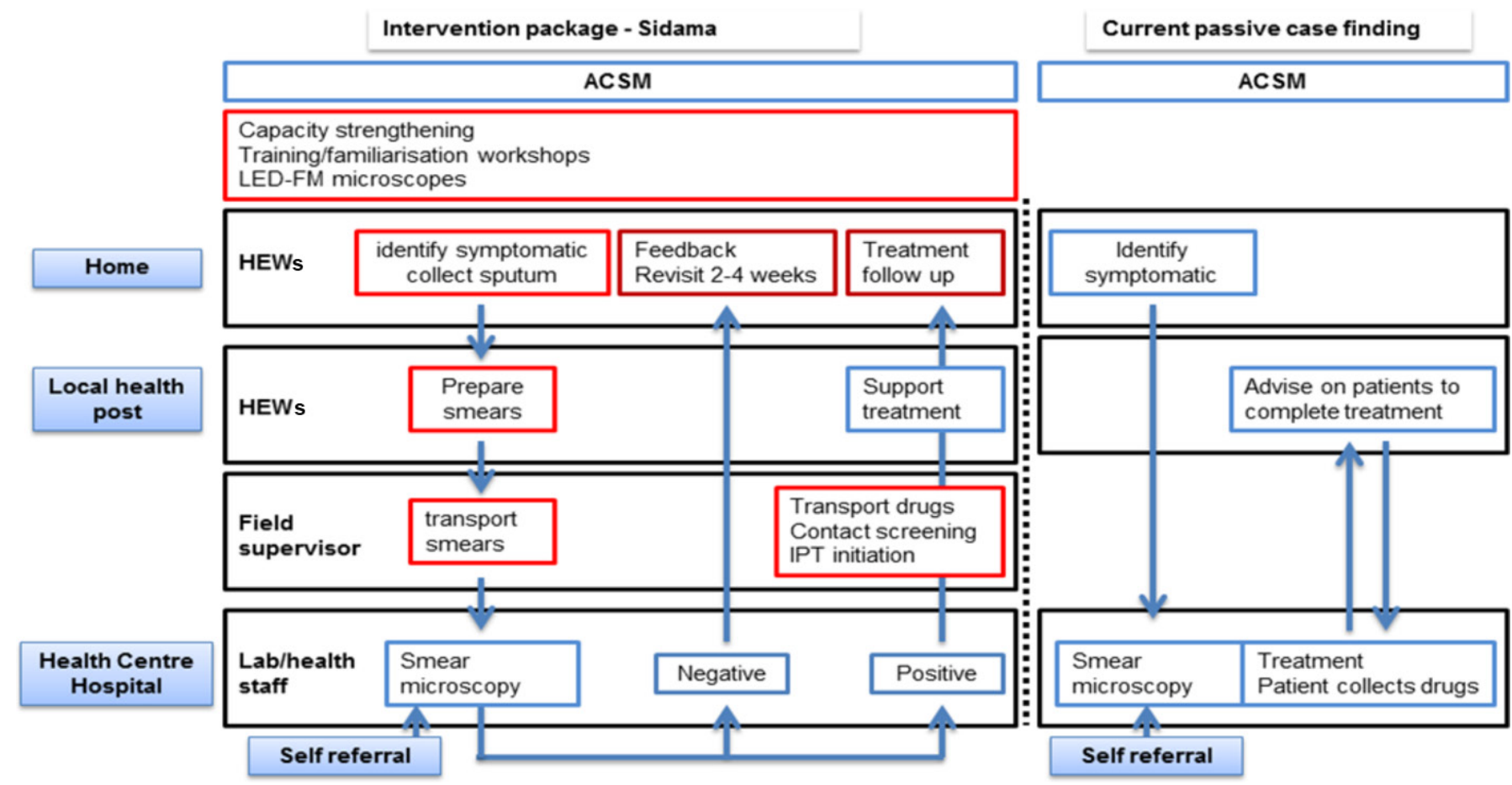

Figure 2 Schematic representation of the intervention package and the current passive case finding approach. ACSM, advocacy, communication and social mobilisation; HEW, health extension worker; IPT, isoniazid preventative therapy; LED-FM, light-emitting diode fluorescence microscopy.

visited at home by the field supervisor and offered initiation of anti-TB treatment. Treatment could be received in the home or at the local health post. Other household members were then screened for TB-associated symptoms and children under 5 years without symptoms were offered isoniazid preventive therapy for 6 months. The HEWs subsequently provided adherence support for the duration of treatment.

As part of the strengthening of the TB laboratories in preparation for the project, 19 light-emitting diode microscopes and two 4-module GeneXpert machines were installed in the diagnostic centres serving the area.

A second zone (Hadiya) with similar characteristics but no geographical connection to Sidama was selected as a control area. Hadiya has a population of about 1.2 million over an area of $3593.31 \mathrm{~km}^{2}$, has similar density of health facilities and treatment services, and has similar socio-economic indicators. This zone was selected because it was considered to resemble the characteristics of the intervention area.

Individuals with $\mathrm{TB}$ identified by the HEWs in the intervention area were registered by proxy by the supervisors in the TB treatment centres nearest to their kebele. Data on the number of TB cases identified by the HEWs and of those self-referring to diagnostic and treatment centres were collected prospectively by the field supervisors on paper case-record forms. Data from the control zone were collected from the routine surveillance quarterly reports and used for secondary data analysis. Only data essential to health services were collected.
TB surveillance and cohort data for Sidama and Hadiya Zones were collected for the 3 years preceding the intervention to establish baseline trends. The quality of the surveillance reports was examined by collecting the data from the original health centre $\mathrm{TB}$ registers and comparing the tallies produced by the project staff with those produced routinely and discrepancies or data duplication were corrected. Health facilities in Sidama (the intervention zone) had tested 12800 symptomatic individuals for TB with smear microscopy in the 12 months prior to the intervention $(1067 /$ month). Of these, $2534(19.8 \%)$ were new smear-positive and 3968 all forms of TB (means 211 and 330 cases/month, respectively), resulting in a case notification rate (CNR) of 64 smear-positive and 100 all forms of TB cases per 100000 population per year prior to intervention. The preintervention baseline male:female ratio of smear-positive cases was 1.3:1. The treatment success rate (TSR) was $76 \%$, with $21 \%$ of persons who started anti-TB treatment being lost to follow-up (LTFU). Health facilities in the control zone (Hadiya) notified 949 smear-positive and 2497 all forms of TB cases (79 and 208 cases/month, respectively) during the same period, resulting in smear-positive and all forms of TB CNRs of 67 and 177 per 100000 population, respectively. The TSR was $82 \%$, with $16 \%$ being LTFU. Prospective TB surveillance and cohort data for Sidama were then collected using the same forms. All patients identified by the HEWs were registered by the health extension supervisors at the nearest health centre providing TB treatment. HEW supervisors also maintained a duplicate 
$\mathrm{TB}$ register to be able to classify patients identified by the intervention and those who attended the health centres. Prospective quarterly reports were also checked for data errors and duplication.

\section{Statistical analysis}

All data were entered into a dedicated database and analysed using SPSS Version 11. Impact was assessed by comparing the annual number of cases detected and treatment outcomes before and after implementation and between the intervention and control zones. Trends in case notifications were calculated before and after the intervention and the reduction in the number of cases was calculated using the first year of the intervention as the baseline. Descriptive data were analysed using summary statistics and $95 \%$ CIs. $\chi^{2}$ tests were used to test differences in proportions over the study period. Continuous variables with normal distributions were compared using means and SD and tested using parametric statistics. p Values $<0.05$ were considered statistically significant.

\section{Ethical considerations}

Support for the implementation of the project was obtained from the Ministry of Health of Ethiopia and the Southern Region Health Bureau.

\section{RESULTS}

\section{Identification of symptomatic individuals and diagnosis of TB}

A total of 216174 (mean 4,003/month) individuals with TB symptoms were identified by the HEWs and a total of 27918 TB cases were registered over the 4.5 intervention years in Sidama Zone. This was equivalent to a mean of 517 TB cases per month, which was higher than the mean of $330 \mathrm{~TB}$ cases per month in the baseline period $(\mathrm{p}<0.001)$. Among the 27918 TB cases, $27468(95.2 \%)$ were new and $1352(4.8 \%)$ retreatment cases.

Sixty-one per cent $(16895 / 27468)$ of the TB cases in the intervention period were smear positive compared with $38 \%(2534 / 6502)$ in the baseline period, with the number of smear-positive cases increasing from a mean of 211 cases per month in the baseline to 312 cases/ month in the intervention period (figure 3). In total, $11130(66 \%)$ of 16895 smear-positive cases were identified by the HEWs through door-to-door visits and 5765 $(34 \%)$ by the health centres. A further 2578 (1.3\%) village participants with smear-negative results received financial support to travel to the clinics to have X-ray examinations. Of these, $1730(67.1 \%)$ were diagnosed as having smear-negative TB based on radiological patterns. X-rays therefore contributed to $6 \%$ of the TB cases notified by the HEWs in the intervention zone.

The smear-positive CNR increased from a baseline of 64 (95\% CI 62.5 to 65.8 ) to 127 (95\% CI 123.8 to 131.2 ) per 100000 population at the start of the intervention and all forms of TB increased from 102 (95\% CI 99.1 to 105.8 ) to 177 (95\% CI 172.6 to 181.0 ) per 100000 population, respectively. There was a peak smear-positive CNR (129 per 100000 population) in the second quarter of the first year of the intervention. The CNR stayed above the baseline after this peak but declined steadily to 80 cases per 100000 population by the end of the intervention (a decline of $9 \%$ per year, $\mathrm{p}<0.01$ ), as shown in figure 3. Likewise, all forms of TB CNR reached 264 per 100000 in the second year of the intervention and declined to 167 (a decline of $37 \%$ ) by the end of the study $(\mathrm{p}<0.01)$.

In comparison, Hadiya reported 2551 smear-positive and 5483 all forms of TB cases during the first 2.5 years when the intervention was implemented in Sidama, resulting in 86 smear-positive and 185 all forms of TB per 100000 population per year, which was similar to the baseline $(\mathrm{p}>0.1)$.

\section{Identification of further cases among household contacts}

A total of $8748(79 \%)$ of the 11130 smear-positive TB cases diagnosed through the HEWs were visited again by the HEW and supervisors to identify household contacts. The visits identified 38534 household contacts, of whom 5737 (14.9\%) had symptoms suggestive of TB. Of these, $4520(78.8 \%)$ produced sputum

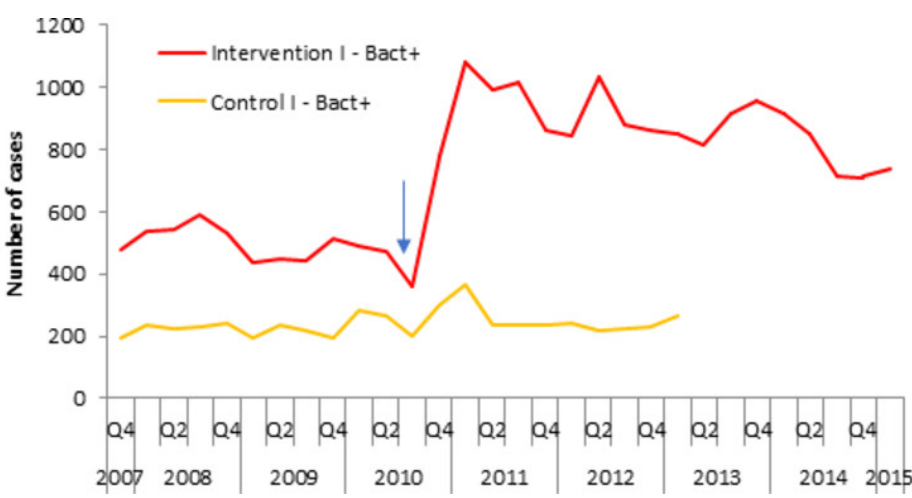

Figure 3 Number of tuberculosis (TB) cases (all forms and bacteriologically positive (Bact+)) detected before and during the intervention in the intervention and control zones. The arrows denote the start of the intervention. 
for examination, resulting in $288(6.4 \%)$ additional smear-positive cases.

\section{Age and gender distribution of patients}

Fifty-eight per cent (125 376) of the 216174 individuals with symptoms of TB identified by the HEWs were female and 90798 (42\%) were male. Women were less likely to be smear positive than men (4\% vs $6 \%$ ) but their larger number resulted in a higher number confirmed to be smear positive (5899 (53\%) female vs 5213 (47\%) male, $\mathrm{p}<0.001)$. The proportion of male-to-female cases changed from 1.3:1 before the intervention to 1:1 during the intervention.

The mean (SD) age of TB cases was 30 (17) years, with $1215(8 \%)$ being $<15$. Male cases were older than female cases (mean (SD) 31 (16) and 29 (14), respectively, $\mathrm{p}<0.001)$. The number of smear-positive, smear-negative and extrapulmonary TB cases by age during the intervention period is shown in table 1 . As expected, most TB cases $<5$ years old were classified as smear-negative or extrapulmonary TB. The proportion of cases classified as smear positive decreased from $65 \%$ in 2010 (779 out of 1198 cases) to $50 \%$ (868 out of 1747) in 2015 ( $\chi^{2}$ for trend, $\mathrm{p}<0.01)$.

\section{Treatment outcome}

Treatment outcome was known for 20108 registered patients in the intervention zone at the time of analysis. The TSR for all forms of TB was $76 \%$ before the intervention (2358/3110) and 95\% (19 003/20 108) during the intervention $(\mathrm{p}<0.001)$, as shown in table 2 . Women had similar treatment outcome as men $(95 \%$ vs $94 \%$ for men). Treatment success was highest among patients 15-35years old and then declined slightly. Children aged $<5$ years and patients aged $>65$ years had relatively low TSRs (91\% and $89 \%$, respectively) than 5-64year-old patients $(\mathrm{p}=0.02$ and $\mathrm{p}<0.001$, respectively) as shown in figure 4 . The proportion of patients LTFU decreased from $651 / 3110(21 \%, 95 \%$ CI $19.5 \%$ to $22.4 \%)$ before the intervention to 598/20 108 (3\%, 95\% CI $2.7 \%$ to $3.2 \%)$ after the intervention $(\mathrm{p}<0.001)$.

\section{DISCUSSION}

Our results achieved over a 4.5-year time span demonstrate that basic diagnostic and treatment support TB services added to the workload of community health workers visiting households door-to-door as part of routine health support can dramatically increase the number of TB cases detected and improve uptake and completion of treatment to over $95 \%$. Moreover, these effects occur rapidly, within a year of implementation, and they can be sustained on a large scale and over a long term.

These results demonstrate that initiatives and tools that address barriers to access diagnosis have enormous potential to improve stagnating case detection rates and could play a significant role to meet the targets of the

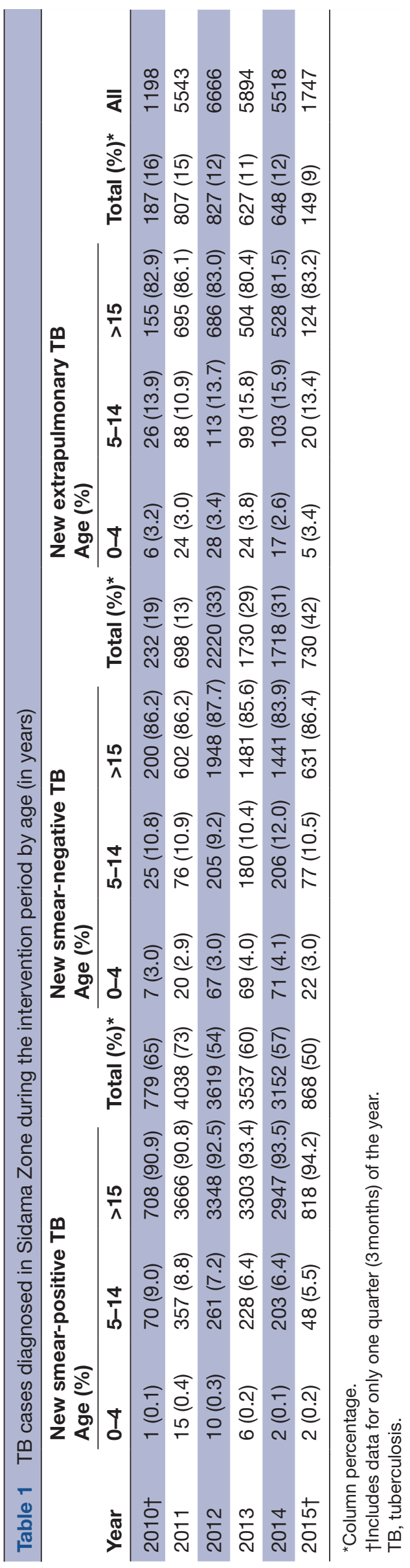


Table 2 Treatment outcome of cohorts registered 1 year prior to the project period and during the implementation period

\begin{tabular}{|c|c|c|c|c|c|c|c|}
\hline \multirow[b]{2}{*}{ Category } & \multicolumn{7}{|c|}{ Treatment outcome, n (\%) } \\
\hline & Cured & Completed & $\begin{array}{l}\text { Treatment } \\
\text { success }\end{array}$ & Died & Failure & $\begin{array}{l}\text { Lost to follow- } \\
\text { up* }\end{array}$ & Registered \\
\hline \multicolumn{8}{|l|}{ Baseline period } \\
\hline New TB cases & 810 & 1429 & $2239(76)$ & $80(2.7)$ & $8(0.3)$ & $608(21)$ & 2935 \\
\hline Retreatment cases & 56 & 63 & $119(68)$ & $13(7)$ & 0 & $43(25)$ & 175 \\
\hline Total & 866 & 1492 & $2358(76)$ & $93(3)$ & $80(3)$ & $651(21)$ & 3110 \\
\hline \multicolumn{8}{|l|}{ Intervention period } \\
\hline New TB cases & 11574 & 7044 & $18618(95)$ & $450(2)$ & $38(0.2)$ & $578(3)$ & 19684 \\
\hline Retreatment cases & 243 & 142 & $385(91)$ & $14(3)$ & $5(1)$ & $20(5)$ & 424 \\
\hline Total & 11817 & 7186 & 19003 (95) & $464(2)$ & $43(0.2)$ & $598(3)$ & 20108 \\
\hline
\end{tabular}

*Includes those patients whose treatment outcome was not available, transferred out, or not evaluated.

TB, tuberculosis.

End TB Strategy. ${ }^{2} 10$ Furthermore, after the initial peak of cases, the number of cases declined substantially, with a mean CNR reduction of $9 \%$ per year, with a staggering $38 \%$ overall reduction in the number of cases between the start and the end of the study at 4.5 years. This is the first large-scale study of its kind to show dramatic increases in TB case detection and the first intervention to achieve such high and sustained rates of TB treatment success, and together with other smaller studies, add to the body of evidence. ${ }^{10}$

The percentage reduction we achieved is the highest reduction reported by any intervention study on the continent, approaching the decline rates achieved in other countries during the Directly Observed Therapy Strategy (DOTS) expansion era, ${ }^{11}{ }^{12}$ when there was a massive focus placed on enhancing TB control and providing consistent diagnostic and treatment services for TB. We show that a simple enhancement of routine community-based care has a massive effect on top of what was achieved by the DOTS expansion. Reductions of a magnitude of $10 \%$ are needed to achieve the international targets of reduction in TB incidence by $2035^{2}$ and undoubtedly, meeting the targets will require further

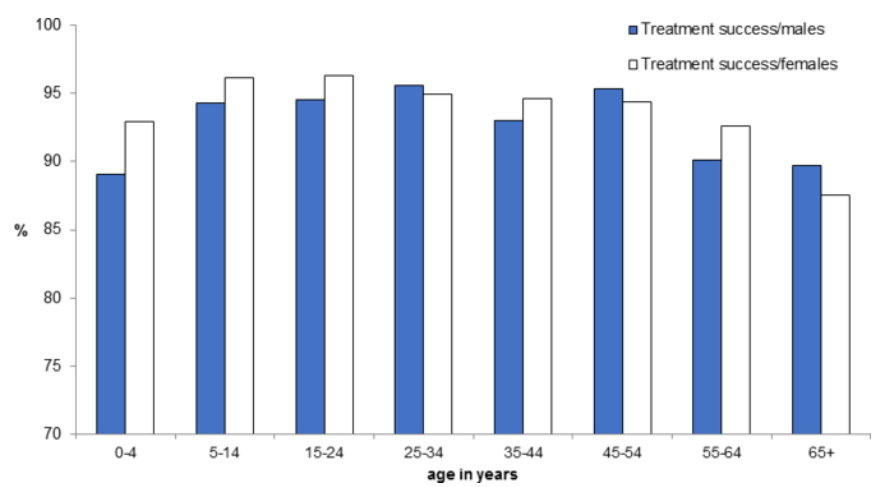

Figure 4 Treatment success rate (cured and treatment completed) of patients with new tuberculosis (TB) treated in intervention zone by gender and age. decentralisation, outreach services and a shift in the mindset of control programmes.

There are multiple intersecting barriers that limit the population's access to TB services, including low disease awareness, stigma, and geographic, gender and socioeconomic barriers. ${ }^{1314}$ These barriers are especially prominent among rural areas which often have the weakest and most poorly staffed health services and patients are too often missed by the way services are currently provided. ${ }^{1516}$ We have thus demonstrated that bringing simple services that detect disease and provide treatment support close to where patients live is critical to increase access to TB diagnosis and treatment adherence and minimise the number of patients LTFU. Indeed, the reduction of LTFU from a high $21 \%$ before the intervention to a minimal $3 \%$ after the intervention is one of the major intervention successes, highlighting that cases that are LTFU may experience important but unquantified barriers accessing services.

Health services should be universal and serve all populations and ideally new interventions should be integrated within existing health system structures to ensure sustainability. Approaches that also facilitate task shifting of services to the locations where they are most needed can strengthen the ability of the National Tuberculosis Programme (NTP) to identify individuals out of reach of stationary health services. Similar task shifting has successfully been used for malaria and HIV ${ }^{17}$ and their positioning within existing community-based structures supports access and facilitates integration into routine services. ${ }^{17-19}$ It is thus likely individuals with other conditions that require access to health services would similarly benefit from locally available services that provide accessible diagnosis and treatment at low cost to the patient. Our intervention has highlighted the large unmet need for services for individuals with undiagnosed TB, but it is likely the same situation occurs for other conditions such as HIV, diabetes and hypertension. Although HEWs used to refer patients with presumptive TB to stationary 
services as part of the HEP package, the number of individuals following their advice was minimal and their ability to diagnose and treat individuals locally increases their efficacy. It is thus likely a multipurpose service that brings the diagnosis and treatment close to the population would increase the efficacy of the HEP.

It is noticeable that among the 27918 TB cases diagnosed, only 1352 (4.8\%) were individuals who had previously received treatment (retreatment cases). This is a low percentage compared with the proportion of retreatment cases reported by the health facilities, suggesting that individuals were less likely to be diagnosed and receive treatment before the services were provided locally by the community health workers. We also observed a change in the gender distribution of cases from 1.3:1 male-tofemale ratio in the health centres before the intervention to $1: 1$ during the intervention. This change may have resulted from the higher proportion of women accessing the service in the villages. While HEWs identified more female than male patients with $\mathrm{TB}$, health centres continued to identify more men than women with TB. This could be due to women facing more difficulties accessing health services for $\mathrm{TB}^{2021}$ or that services delivered by female HEWs within the village facilitate reaching women more readily than men. ${ }^{22} 23$

Our findings need to be interpreted within the limitations of the study. Although the increased detection and subsequent reduction of cases could be due to improved access to diagnosis, the identification of a backlog of cases and subsequent reduction of transmission, the study design does not allow us to establish causality and it could be equally due to staff fatigue and poor performance of the programme. Its non-randomised and open design is inferior to a randomised trial and concurrent interventions in the area may have led to confounding and misinterpretation of data. The package was also less efficient confirming children, as children are rarely able to provide sputum. X-rays and Xpert were used by a small proportion of participants but this required attending health facilities and further childfriendly approaches are needed. ${ }^{24-27}$ Furthermore, the package was intentionally comprehensive and integrated and it is difficult to single out which components have more impact than others. NTP staff however often discuss whether some components are more efficient than others and want to cherry-pick the cheaper components or those perceived to be most important. Finally, despite the significant improvement in TB treatment outcome, some age groups continued to have slightly lower TSRs than others. This is especially true for young children and older individuals above 65 years old. There may be further biological and social factors that increase the likelihood of unfavourable treatment outcome among these age groups.

Our findings demonstrate that high case notification and treatment success could be achieved within the existing HEP in rural communities of Ethiopia. The strength of the intervention was likely due to its embeddedness within the existing HEP and the NTP; its partnership with a functioning HEW system, strategic engagement with stakeholders and its alignment with national health policies and priorities. We created evidence to inform decision-making and policy changes at national scale and the NTP recently included the package in its request for funding from the Global Fund. Funds to scale up the intervention throughout the country were awarded and the intervention is being scaled up to other zones.

In conclusion, the service engaged the community, contributed to health system strengthening and provided a continuum of care, resulting in significant improvements in both case finding and treatment outcome. A key component of the package was its integration with the HEP and the NTP which facilitated sustainability. Interventions that are integrated into existing health systems and community-based approaches can contribute to the delivery of universal and equitable $\mathrm{TB}$ diagnostic and treatment services for all populations, including those who are vulnerable or disadvantaged. With the onus on universal healthcare and the Sustainable Development Goals (SDGs) there is increasing interest in community-based health systems and community health workers. ${ }^{28}$ Country contexts where these are well established ${ }^{29}$ could consider similar approaches, as the availability of a functioning community system would facilitate the implementation and sustainability of the package.

Acknowledgements We are thankful to the Federal Ministry of Health of Ethiopia, the Southern Nations, Nationalities, and Peoples' Regional Health Bureau, the Sidama Zone Health Department, and the district health offices, field supervisors, health workers, health extension workers and community for their support in the implementation of this project.

Contributors The study was conceived by DGD, MAY and LEC who, together with SJT, were alternating principal investigators over the years on behalf of REACH Ethiopia and the Liverpool School of Tropical Medicine. This was due to MAY, senior research fellow in Liverpool, moving to the Global Fund, and LEC moving to (and then returning from) TDR/WHO Geneva over 2 years. MAY and LEC developed the concept of the study and together with SJT prepared the initial protocol in 2009. DGD was the project director in Ethiopia. LB, SS and JC advised on the initial design of the intervention and, with MAY, on how to contextualise the study on its international policy relevance. The same authors provided technical support over the 5 years, high level advocacy, linkage to policymakers, and support to engage and influence the government supporting the upscale of the service and policy guidance. Data collection was led by DGD; data analysis and interpretation was conducted by DGD, LEC and MAY; SJT supported the interpretations on gender, equity and sustainability and led qualitative studies that supplemented the study. LEC, DGD, SJT and MAY wrote the first draft of the manuscript. All authors contributed to the final manuscript.

Funding The project was funded by the Global Affairs Canada, through the TB REACH Initiative of the Stop TB Partnership, Waves I and III project numbers T9-370-114 ETH and GAL W3/2013, and a grant from the UK Economic and Social Research Council (ESRC): grant reference ES/L007746/1. The funders played no role in the decision to publish or the interpretation of the data.

Competing interests SS and JC work at the Stop TB Partnership and coordinated the proposal review committee which makes funding allocations for TB REACH, but did not participate in the funding decisions. Their contribution focused on sharing lessons learnt from similar TB REACH projects, the contextualisation of the project within the international initiatives, suggesting ways the project could improve performance and engaging international stakeholders to promote support for the project. LB was the independent monitoring and evaluation expert and interpreted surveillance and the project's databases over the project lifetime. She reported to TB REACH on project performance. Final decisions on project implementation were taken by DGD, MAY, SJT and LEC

Ethics approval Ethical approval was obtained from the Research Ethics Committee of the Liverpool School of Tropical Medicine, UK (LSTM, protocol number 10.69). 
Provenance and peer review Not commissioned; externally peer reviewed.

Data sharing statement The authors can share the databases upon request.

Open Access This is an Open Access article distributed in accordance with the Creative Commons Attribution Non Commercial (CC BY-NC 4.0) license, which permits others to distribute, remix, adapt, build upon this work non-commercially, and license their derivative works on different terms, provided the original work is properly cited and the use is non-commercial. See: http://creativecommons.org/ licenses/by-nc/4.0/

(C) Article author(s) (or their employer(s) unless otherwise stated in the text of the article) 2017. All rights reserved. No commercial use is permitted unless otherwise expressly granted.

\section{REFERENCES}

1. WHO. WHO Global report on diabetes. Geneva, 2016. doi:/entity/ diabetes/global-report/en/index.html.

2. Partnership ST. The Global Plan to End TB. Geneva: WHO, 2015.

3. Zhou C, Long Q, Chen J, et al. Factors that determine catastrophic expenditure for tuberculosis care: a patient survey in China. Infect Dis Poverty 2016;5:6.

4. Dye C, Maher D, Weil D, et al. Targets for global tuberculosis control. Int J Tuberc Lung Dis 2006;10:460-2.

5. Styblo K, Bumgarner JR. Tuberculosis can be controlled with existing technologies: Evidence. The Hague: Tuberculosis Surveillance Research Unit, 1991.

6. Cambanis A, Yassin MA, Ramsay A, et al. Rural poverty and delayed presentation to tuberculosis services in Ethiopia. Trop Med Int Health 2005;10:330-5.

7. Tanimura T, Jaramillo E, Weil D, et al. Financial burden for tuberculosis patients in low- and middle-income countries: a systematic review. Eur Respir J 2014;43:1763-75.

8. WHO. Global Tuberculosis Report 2015 Geneva: WHO; 2015 [WHO/ $\mathrm{HTM} / \mathrm{TB} / 2015.22:[T h i s$ is the twentieth global report on tuberculosis (TB) published by WHO in a series that started in 1997. It provides a comprehensive and up-to-date assessment of the TB epidemic and progress in implementing and financing TB prevention, care, control and research at global, regional and country levels using data reported by over 200 countries that account for more than 99\% of the world's TB cases. In this 2015 edition, particular attention is given to assessment of whether 15 global TB targets set in the context of the Millennium Development Goals were achieved worldwide and at regional and country levels. 2016 http://www.who. int/tb/publications/global_report/en/ (accessed 4 Jan 2016).

9. Yassin MA, Datiko DG, Tulloch O, et al. Innovative community-based approaches doubled tuberculosis case notification and improve treatment outcome in Southern Ethiopia. PLoS One 2013;8:e63174.

10. Creswell J, Sahu S, Blok L, et al. A multi-site evaluation of innovative approaches to increase tuberculosis case notification: summary results. PLoS One 2014;9:e94465.

11. Suárez PG, Watt CJ, Alarcón E, et al. The dynamics of tuberculosis in response to 10 years of intensive control effort in Peru. $J$ Infect Dis 2001;184:473-8.
12. China Tuberculosis Control Collaboration. The effect of tuberculosis control in China. Lancet 2004;364:417-22

13. Tulenko K, Møgedal S, Afzal MM, et al. Community health workers for universal health-care coverage: from fragmentation to synergy. Bull World Health Organ 2013;91:847-52.

14. UNICEF. Access to healthcare through community health workers in East and Southern Africa 2014. 2016 http://www.unicef.org/health/ files/Access_to_healthcare_through_community_health_workers_in_ East_and_Southern_Africa.pdf (accessed 15 Feb 2016).

15. Saunders MJ, Evans CA. Fighting poverty to prevent tuberculosis. Lancet Infect Dis 2016;16:395-6.

16. Barter DM, Agboola SO, Murray MB, et al. Tuberculosis and poverty: the contribution of patient costs in sub-Saharan Africa--a systematic review. BMC Public Health 2012;12:980.

17. Zachariah R, Ford N, Philips M, et al. Task shifting in HIV/AIDS: opportunities, challenges and proposed actions for sub-Saharan Africa. Trans R Soc Trop Med Hyg 2009;103:549-58.

18. Crisp N, Chen L. Global supply of health professionals. N Engl J Med 2014;370:950-7.

19. Emdin CA, Chong NJ, Millson PE. Non-physician clinician provided HIV treatment results in equivalent outcomes as physician-provided care: a meta-analysis. J Int AIDS Soc 2013;16:18445.

20. Thorson A, Johansson E. Equality or equity in health care access: a qualitative study of doctors' explanations to a longer doctor's delay among female TB patients in Vietnam. Health Policy 2004;68:37-46.

21. Bashour H, Mamaree F. Gender differences and tuberculosis in the Syrian Arab Republic: patients' attitudes, compliance and outcomes. East Mediterr Health J 2003;9:757-68.

22. Datiko DG, Yassin MA, Tulloch O, et al. Exploring providers' perspectives of a community based TB approach in Southern Ethiopia: implication for community based approaches. BMC Health Serv Res 2015;15:501.

23. Tulloch O, Theobald S, Morishita F, et al. Patient and community experiences of tuberculosis diagnosis and care within a communitybased intervention in Ethiopia: a qualitative study. BMC Public Health 2015;15:187.

24. Dangisso MH, Datiko DG, Lindtjørn B. Low case notification rates of childhood tuberculosis in southern Ethiopia. BMC Pediatr 2015;15:142.

25. Elhassan MM, Elmekki MA, Osman AL, et al. Challenges in diagnosing tuberculosis in children: a comparative study from Sudan. Int J Infect Dis 2016;43:25-9.

26. Joshi B, Chinnakali $P$, Shrestha A, et al. Impact of intensified casefinding strategies on childhood TB case registration in Nepal. Public Health Action 2015;5:93-8.

27. Weaver MS, Lönnroth K, Howard SC, et al. Interventions to improve adherence to treatment for paediatric tuberculosis in low- and middle-income countries: a systematic review and meta-analysis. Bull World Health Organ 2015;93:700-11.

28. Theobald S, Hawkins $\mathrm{K}$, Kok M, et al. Close-to-community providers of health care: increasing evidence of how to bridge community and health systems. Hum Resour Health 2016;14:32.

29. Ramsey K, Hingora A, Kante M, et al. The Tanzania Connect Project: a cluster-randomized trial of the child survival impact of adding paid community health workers to an existing facility-focused health system. BMC Health Serv Res 2013;13(Suppl 2):S6. 